OPEN ACCESS

Edited by:

Catherine Ropert,

Federal University of Minas Gerais,

Brazil

Reviewed by:

Fouzia Sadiq,

Shifa Tameer-e-Millat University,

Pakistan

Humberto Gravina,

University of São Paulo, Brazil

*Correspondence:

Lei Zhang

drzhanglei@/zu.edu.cn

Xue-Li Bai

shirleybai@zju.edu.cn

Specialty section:

This article was submitted to Microbial Immunology,

a section of the journal

Frontiers in Microbiology

Received: 26 August 2021 Accepted: 05 October 2021 Published: 02 November 2021

Citation:

He L-H, Yao D-H, Wang L-Y,

Zhang $L$ and Bai $X-L$ (2021) Gut Microbiome-Mediated Alteration of

Immunity, Inflammation, and Metabolism Involved in the Regulation of Non-alcoholic Fatty Liver Disease.

Front. Microbiol. 12:761836.

doi: 10.3389/fmich.2021.761836

\section{Gut Microbiome-Mediated Alteration of Immunity, Inflammation, and Metabolism Involved in the Regulation of Non-alcoholic Fatty Liver Disease}

\author{
Li-Hong He ${ }^{1,2}$, Dun-Han Yao ${ }^{2}$, Ling-Yun Wang ${ }^{2}$, Lei Zhang ${ }^{2 *}$ and Xue-Li Bai ${ }^{1 *}$ \\ 'Department of Hepatobiliary and Pancreatic Surgery, the First Affiliated Hospital, School of Medicine, Zhejiang University, \\ Hangzhou, China, ${ }^{2}$ The First Clinical Medical College, Lanzhou University, Department of General Surgery, The First Hospital \\ of Lanzhou University, Lanzhou, China
}

Non-alcoholic fatty liver disease (NAFLD) is one of the leading causes of end-stage liver disease, leading to a rapidly growing global public health burden. The term "gut microbiome (GM)" refers to the approximately 100 trillion microbial cells that inhabit the host's gastrointestinal tract. There is increasing evidence that $\mathrm{GM}$ is involved in the pathogenesis of NAFLD and may be a potential target for intervention. To explore GM-based strategies for precise diagnosis and treatment of NAFLD, great efforts have been made to develop a comprehensive and in-depth understanding of the host-microbe interaction. This review evaluates this interaction critically, mainly considering the intricate regulation of the metabolism, immunity, and inflammatory status during the evolution of the disease pathogenesis, revealing roles for the GM in NAFLD by examining advances in potential mechanisms, diagnostics, and modulation strategies.

Synopsis: Considering the intricate metabolic and immune/inflammatory homeostasis regulation, we evaluate the latest understanding of the host-microbe interaction and reveal roles for the gastrointestinal microbiome in NAFLD. Strategies targeting the gastrointestinal microbiome for the diagnosis and treatment of NAFLD are proposed.

Keywords: gut microbiota, non-alcoholic fatty liver disease, immune, metabolism, gut-liver axis

\section{INTRODUCTION}

Non-alcoholic fatty liver disease (NAFLD) describes a collection of hepatic clinicopathological syndromes that range from simple hepatic steatosis, non-alcoholic steatohepatitis (NASH) to fat-related fibrosis and cirrhosis (Brunt et al., 2015). Characterized by excessive fat accumulation without a definite liver damaging factor, NAFLD is an acquired metabolic stress liver injury closely related to obesity, insulin resistance, and genetic susceptibility (Brunt et al., 2015; Wang and Malhi, 2018). Resulting from continuous damage to hepatocytes, the incidence of hepatocellular carcinoma (HCC) in patients with NAFLD is much higher than that in healthy people (Ipsen et al., 2018; Younossi et al., 2019). In some western countries, NAFLD has 
become the fastest-growing cause of HCC (Huang et al., 2021). With the prevalence of obesity and metabolic syndrome, NAFLD has become a major chronic liver disease worldwide, causing a global public health concern (Younossi et al., 2016; Huang et al., 2021). Considering its high morbidity, poor prognosis, and the lack of targeted and effective drugs, strategies to prevent and treat NAFLD are urgently needed to reduce the increasing burden of the disease.

The human gastrointestinal microbiome (GM) refers to the assemblage of microorganisms (e.g., bacteria, fungi, viruses, and protozoans) that inhabit the gastrointestinal tract (Sender et al., 2016). The results of metagenomic sequencing indicated that the GM comprises more than 1,000 kinds of microorganisms, affected by genetics, eating habits, and environmental factors (Zhernakova et al., 2016). A balanced GM plays a beneficial role in the physiological regulation of the host by balancing local and systemic immune responses, maintaining normal gut-liver circulation, and inhibiting pathogen colonization. Dysbacteriosis will lead to various diseases (e.g., metabolic diseases, immune diseases, respiratory diseases, and even tumors; Hand et al., 2016; Gong et al., 2018; Schirmer et al., 2018; Canfora et al., 2019; He et al., 2020). In particular, considerable research has demonstrated that the GM and its metabolites potentially affect the occurrence and prognosis of NAFLD by participating in the host's immune and inflammatory responses, and nutrient intake and metabolism (Aron-Wisnewsky et al., 2020; Hu et al., 2020). Similarly, dysbacteriosis and the resulting increased gut inflammation and weakened immune surveillance play pivotal roles in leading to NASH, cirrhosis, and NAFLD-related HCC (Tripathi et al., 2018; Ezzaidi et al., 2019; Albillos et al., 2020).

In the present review, we dissect the role of the GM and their inflammatory mediators on immune regulation in NAFLD. Specifically, we focus on the characteristic changes of the GM in patients with NAFLD, including diversity and uniformity/ homogeneity, and the developed non-invasive diagnostic strategies. In addition, the mechanism by which the GM regulates metabolic and immune homeostasis during the onset and progression of NAFLD and advances in modulating the GM to treat NAFLD are also highlighted.

\section{THE GUT-LIVER AXIS AND THE INTESTINAL BARRIER}

Many studies have demonstrated cross-talk between the GM and multiple organs of the host, which affects local and systemic metabolism and immune homeostasis (Hand et al., 2016; Gong et al., 2018; Schirmer et al., 2018; Canfora et al., 2019; He et al., 2020). The interaction among the gut, its contents, and the liver is called the "gut-liver axis," resulting from the

\footnotetext{
Abbreviations: GM, Gastrointestinal microbiome; NAFLD, Non-alcoholic fatty liver disease; NASH, Non-alcoholic steatohepatitis; HCC, Hepatocellular carcinoma; BAs, Bile acids; SCFAs, Short chain fatty acids; LPS, Lipopolysaccharide; HFD, High fat diet; TLRs, Toll-like receptors; NLRP3, NOD-like receptor family, pyrin domain containing 3; GLP, Glucagon-like peptide; TGR5, Takeda G-protein-coupled receptor 5; TMA, Trimethylamine; TMAO, Trimethylamine-N-oxide.
}

integrated signals generated by genes, diet, and environmental factors (Figure 1A; Tripathi et al., 2018). The portal vein and biliary system are the basis of this bidirectional interaction. On the one hand, the portal vein can transport intestinal origin immune cells, cytokines, and gut-derived products directly to the liver, such as secondary bile acids (BAs), short chain fatty acids (SCFAs), and lipopolysaccharide (LPS). On the other hand, the liver can secrete bile and many bioactive mediators into the intestine through the biliary system (Tripathi et al., 2018; Albillos et al., 2020). The interdependence between the liver and the gut explains why intestinal barrier damage can lead to some components of the microbiota and their metabolites flowing into the liver, leading to or aggravating a series of liver diseases.

The intestinal barrier in the gut-liver axis includes physical, immune, and biochemical components, plays an important role in the gut-liver axis (Peterson and Artis, 2014). The gut vascular and single layer epithelial cells, linked by tight junction proteins, together with the mucus layer and microorganisms, constitute a physical barrier. Molecules with antimicrobial properties, such as BAs and antimicrobial proteins, maintain and mediate biochemical barriers. Secreted immunoglobulin A (SIgA) and lymphoid follicles containing a variety of immune cells are the main parts of the immune barrier. In the normal physiological state, the intestinal barrier constitutes the first line of defense in human immunity, while the liver provides the second line of defense for pathogenic factors that escape from the intestinal mucosal immune defense; the immune tissues in the intestine and liver participate in the immune tolerance to food antigens and the clearance of pathogens (Martens et al., 2018).

Gastrointestinal microbiome dysbiosis can disrupt these barriers, increasing mucosal permeability. Dietary factors can not only alter the intestinal microbiome composition, but also play a vital role in the maintenance of the intestinal barrier. The pathological state of the intestinal barrier induced by a high fat diet (HFD) results in intestinal bacterial translocation and endotoxin entering the portal venous system (De Santis et al., 2015). As feedback, immune cells in the liver are activated by these pathogenic factors, releasing host inflammatory factors, and resulting in tissular damage to the intestinal mucosa, liver, and systemic organs (Szabo, 2015). The GM and its metabolites have a range of effects on the health and disease of liver, and the methods to promote optimum liver health are a major concern.

\section{INTESTINAL MICROECOLOGY DISORDER IN PATIENTS WITH NAFLD}

The characteristics of the GM in patients have both homogeneity and heterogeneity (Table 1). Wang et al. (2016) observed a lower diversity and a phylum-level change in the GM in patients with NAFLD. Compared with those in the healthy control group, the patients had $20 \%$ more Bacteroidetes and $24 \%$ less Firmicutes. Notably, the abundances of four families of Firmicutes were decreased significantly, including Lachnospiraceae, Ruminococcaceae, Lactobacillaceae, and Peptostreptococcaceae, which are SCFA-producing and $7 \alpha$-dehydroxylating bacteria 


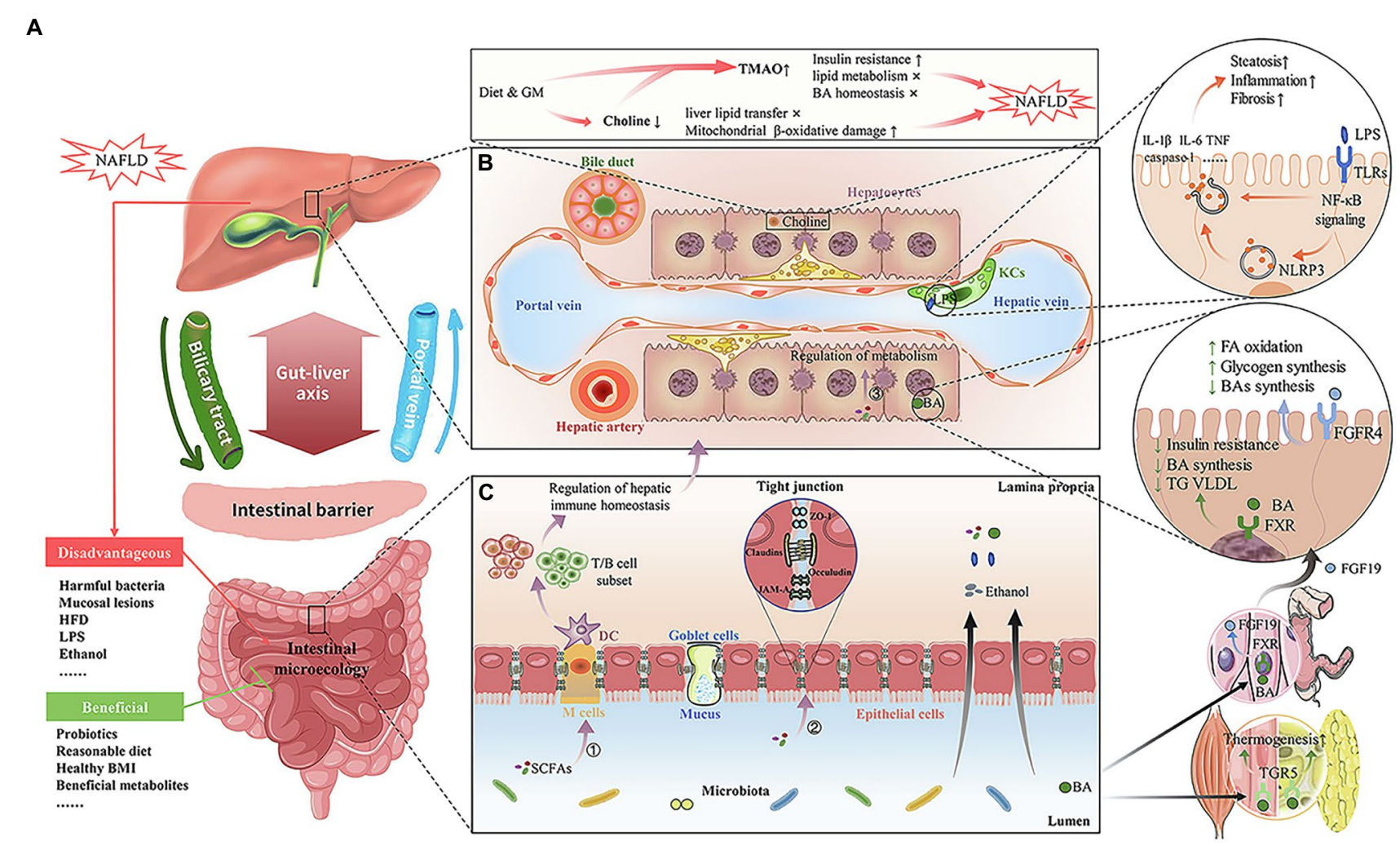

FIGURE 1 | Interaction between the intestinal flora, its metabolites, and Non-alcoholic fatty liver disease (NAFLD). (A) Gut-liver axis. Based on the portal vein and biliary system, there is an interaction between the liver and the gut/gastrointestinal microbiome (GM). A healthy intestinal barrier and intestinal microecology are beneficial to maintain liver health. The disadvantageous factors include NAFLD, harmful bacteria, mucosal lesions, HFD, lipopolysaccharide (LPS), and ethanol. The beneficial factors include probiotics, reasonable diet, healthy BMI, and beneficial metabolites. (B) The liver microenvironment and GM-derived metabolites associated with NAFLD. Choline deficiency and TMAO increase caused by diet and GM disorders can lead to or aggravate NAFLD by enhancing insulin resistance and lipid metabolism disorder. The transfer of excessive LPS, secondary to intestinal barrier damage, to the liver will interact with TLRs on Kupffer cells and stellate cells, thus activating NF-кB and NLRP3 signaling pathways to produce hepatic pro-inflammatory and pro-fibrotic mediators, which aggravates NAFLD. The nuclear receptor, FXR, is activated by BAs in the liver and has several downstream effects, such as inhibition of TG, VLDL, and BA synthesis, and increased insulin sensitivity. BAs also activate TGR5 in muscle and adipose tissues, thereby increasing thermogenesis and energy expenditure. In the terminal ileum, and after BA uptake by the ileal apical sodium-dependent bile acid transporter, FXR also stimulates production of FGF19, which, upon binding to FGFR4 in liver cells, represses BA synthesis and promotes hepatic glycogen storage and FA oxidation. (C) The gut microenvironment and GM-derived metabolites associated with NAFLD. The intestinal barrier, including intestinal epithelial cells and tight junctions, plays an important role in intestinal tract permeability. When the intestinal barrier is destroyed, intestinal permeability will increase, and then the bacteria and their metabolites (e.g., endogenous alcohol) will transfer to the liver and eventually cause or aggravate NAFLD. SCFA is the most important metabolite of intestinal bacteria, which affects liver health in three ways, including immunity, nutrient intakeand metabolism, and tight junction function.

(Wang et al., 2016). In a prospective cross-sectional study, the abundances of Ruminococcus, Paucalinbacterium prausnitzii, and Coprococcus in patients with NAFLD were lower than those in healthy people; the difference was independent of bodymass index and insulin resistance (Da Silva et al., 2018). Interestingly, patients with NAFLD have higher fecal concentrations of propionic acid and isobutyric acid, and higher serum concentrations of 2-hydroxybutyric acid and L-lactic acid, than healthy controls (Da Silva et al., 2018). A cohort study in China showed that $60 \%$ of patients with NAFLD had a high abundance of Klebsiella pneumoniae (alcohol highproducing; Yuan et al., 2019). In the mouse model, a specific K. pneumoniae-rich microbiota isolated from patients with NAFLD and transplanted into healthy mice could aggravate liver inflammation and induce NAFLD (Yuan et al., 2019), which implied endogenous alcohol produced by these bacteria is an important pathogeny of NAFLD.

Most patients with NAFLD are obese but some patients belong to "lean NAFLD," its pathogenesis remaining unclear. A recent study showed that the lean NAFLD group has a more Dorea and total BAs, but a fewer Marvinbryantia and Christensellenaceae R7, compared with lean healthy control, which provides an insight into microbial drivers of lean NAFLD pathogenesis (Younes and Bugianesi, 2019; Chen et al., 2020).

These findings indicated the potential role of specific microbiota and the characteristics of its metabolites in the pathogenesis of NAFLD. Based on these characteristics, the GM might be used as a non-invasive biomarker of NAFLD phenotype and provide prognostic value in the risk of progression to cirrhosis and HCC (Table 2). 
TABLE 1 | Research on the changes of GM and metabolites in patients with NAFLD.

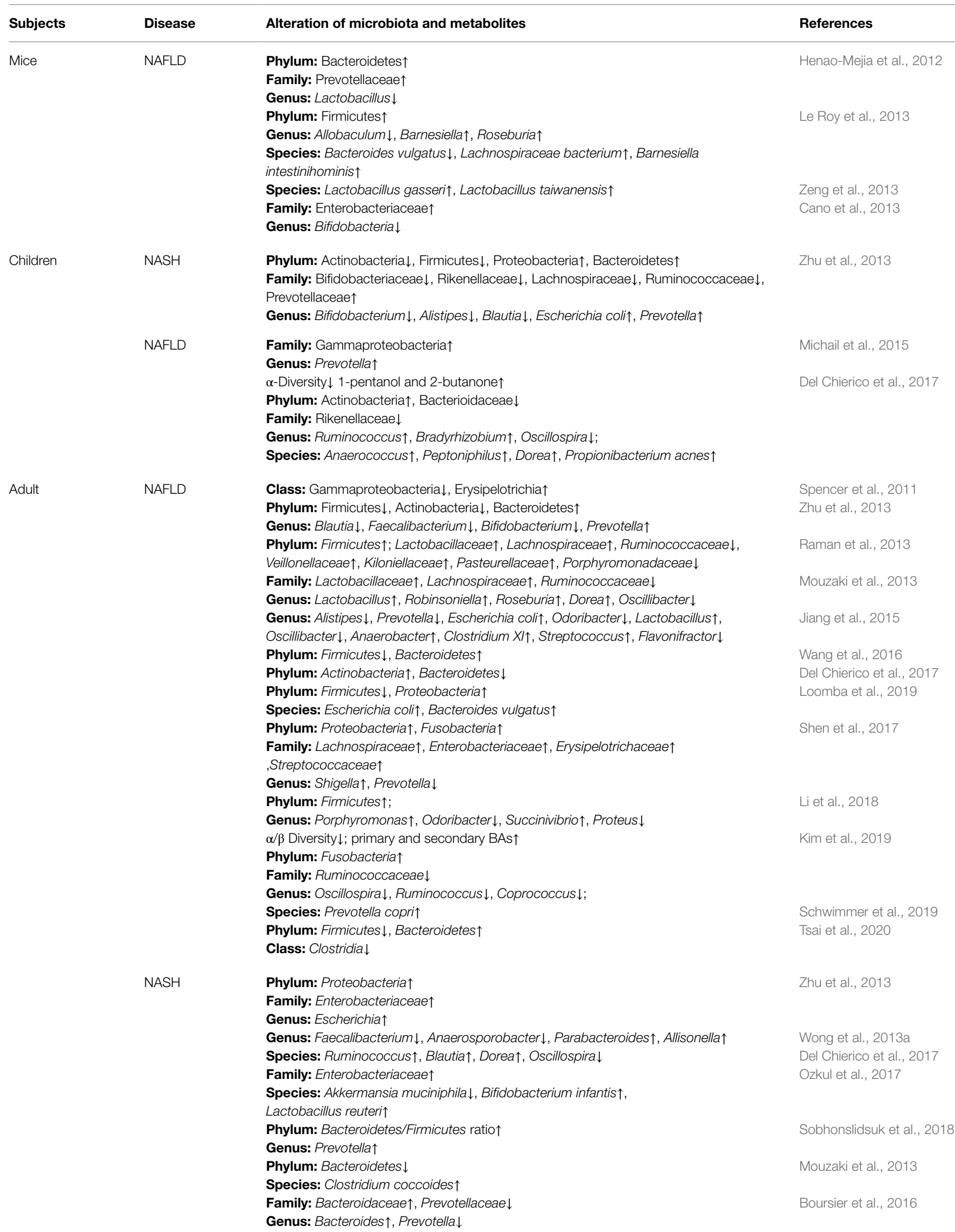




\section{POSSIBLE MECHANISM OF GM'S EFFECT ON THE OCCURRENCE AND PROGNOSIS OF NAFLD}

The pathogenesis of NAFLD is thought to involve complex interactions among genetic susceptibility, environmental factors, insulin resistance, and changes in the GM. The "multiple-hit" hypothesis is adequate to explain the diverse metabolic and molecular changes observed in the development of NAFLD (Buzzetti et al., 2016; Fang et al., 2018). With the progress of metagenomics and non-targeted metabolomics, the role of the GM in the pathogenesis of NAFLD has attracted the attention of the scientific community. The GM plays an important role in the maintenance of host immune and inflammatory homeostasis, and the balance of nutrient intake and metabolism, thus directly or indirectly affecting the onset and development of NAFLD (Figure 1; Chu et al., 2019; Jennison and Byrne, 2021).

\section{Damage to the Intestinal Barrier and Aggravation of Secondary Inflammation}

Emerging evidence shows that an intestinal barrier disorder leads to the translocation of the GM and metabolites, which can reach the liver directly along the gut-liver axis. Patients with NAFLD have decreased expression of junctional adhesion molecule A and zonula occludens-1, and increased intestinal permeability, which might be important factors in disease progression (Kolodziejczyk et al., 2019). Compared with simple hepatic steatosis, the correlation of increased intestinal permeability was stronger in patients with $\mathrm{NASH}$, suggesting that inflammatory persistence and exacerbation might be caused by destruction of the intestinal barrier (Luther et al., 2015).

TABLE 2 | Clinical research on the diagnosis of NAFLD and related diseases by targeting GM.

\begin{tabular}{|c|c|c|}
\hline Disease & $\begin{array}{l}\text { Diagnostic tool and } \\
\text { mechanism }\end{array}$ & References \\
\hline NAFLD-liver fibrosis & $\begin{array}{l}\text { Based on the specific } \\
\text { differences in microbiota and } \\
\text { BAs in both blood and feces } \\
\text { that correlate with the } \\
\text { presence of liver fibrosis }\end{array}$ & Lelouvier et al., 2016 \\
\hline NAFLD-liver fibrosis & $\begin{array}{l}\text { Based on a Random Forest } \\
\text { classifier model containing } 40 \\
\text { features (including } 37 \text { bacterial } \\
\text { species) }\end{array}$ & Loomba et al., 2017 \\
\hline $\begin{array}{l}\text { Hepatic steatosis and } \\
\text { fibrosis }\end{array}$ & $\begin{array}{l}\text { Based on the link between the } \\
\text { abundance of specific GM } \\
\text { and 3-(4-hydroxyphenyl) } \\
\text { lactate that shares a gene } \\
\text { effect with hepatic steatosis } \\
\text { and fibrosis }\end{array}$ & Caussy et al., 2018 \\
\hline NAFLD & $\begin{array}{l}\text { Based on molecular networks } \\
\text { linking the GM and the host } \\
\text { molecular phenomics (hepatic } \\
\text { transcriptome and plasma and } \\
\text { urine metabolomes) to hepatic } \\
\text { steatosis. }\end{array}$ & Hoyles et al., 2018 \\
\hline
\end{tabular}

In a mouse model, compared with the control group, the zonula occludens-1-deficient mouse group (intestinal barrier disorder model) fed a high fat, fructose, and cholesterol diet, developed more severe NASH (Rahman et al., 2016). Similarly, destruction of the intestinal barrier is considered an early event in the pathogenesis of NAFLD. Mice fed an HFD suffered from diet-induced intestinal dysbacteriosis after only 1 week, resulting in intestinal barrier damage and bacterial translocation to the liver (De Santis et al., 2015; Mouries et al., 2019). Thus, an impaired intestinal barrier secondary to dysbacteriosis might be a prerequisite for diet-driven NAFLD.

It is unclear whether NAFLD is the cause or the result of intestinal barrier disruption. However, it can be confirmed that no matter what the triggering event is, the transfer of pro-inflammatory products, such as LPS, secondary to intestinal barrier damage, to the systemic circulation, will aggravate NAFLD and lead to poor prognosis (Gabele et al., 2011; Ipsen et al., 2018; Mouries et al., 2019). As shown in Figure 1B, excessive LPS translocation to the liver can interact with tolllike receptor (TLR)-4 on Kupffer cells and stellate cells, thus activating the nuclear factor kappa $\mathrm{B}(\mathrm{NF}-\mathrm{\kappa B})$ signaling pathway, and ultimately promoting the release of pro-inflammatory cytokines, such as interleukin (IL)-1, IL-6, and tumor necrosis factor, aggravating hepatic steatosis, inflammation, and fibrosis (Henao-Mejia et al., 2012; Plociennikowska et al., 2015; Carpino et al., 2020). TLR signaling in the mucosa also leads to the production of NOD-like receptor family, pyrin domain containing 3 (NLRP3), which results in the production of hepatic pro-inflammatory and pro-fibrotic mediators (e.g., caspase-1, IL-1 $\beta$, and IL-18; Mridha et al., 2017; Carpino et al., 2020).

\section{Regulation of SCFAs in Metabolic and Inflammatory Pathways}

Human SCFAs (e.g., acetate, propionate, and butyrate) are generated mainly from the fermentation of polysaccharides by the GM, and play a pivotal role in energy metabolism and inflammation regulation (Gomes et al., 2018). The different phenotypes of the GM and different dietary factors will affect the type and quantity of SCFAs synthesized in the gut. A high-fiber or resistant starch diet, the Mediterranean diet, and the enrichment of specific bacteria, such as Akkermansia municiphilla (producing propionate), Ruminococcus, Faecalibacterium, and Eubacterium (producing butyrate), can induce SCFA production (Morrison and Preston, 2016; Gomes et al., 2018).

Many polysaccharides cannot be hydrolyzed by the host, but can be realized by specific microbiota, finally generating SCFAs. If the excess SCFAs are not metabolized by colon cells, they will enter the liver and peripheral circulation through the portal vein, where they can be used as the substrates for fat synthesis and glycogenesis (Rau et al., 2018). This enables the host to obtain excess energy from food more efficiently, and to synthesize and store more fat to the liver (Harris et al., 2020). In a cohort study, with the development of NAFLD, higher abundances of SCFA-producing bacteria and intestinal acetate and propionate levels were observed (Rau et al., 2018). Interestingly, elevated peripheral levels of 
pro-inflammatory $\mathrm{T}$ cells (lower numbers of resting regulatory T-cells and higher numbers of Th17 cells) were observed simultaneously, which suggested that SCFAs are involved in the development of NAFLD, not only by affecting metabolism, but also by influencing immune and inflammatory responses (Rau et al., 2018). NAFLD is most associated with obesity. In mouse models and human studies, obese subjects have more carbohydrate metabolism genes in the intestinal microbiome and a higher concentration of SCFAs in the cecum, indicating that their production is excessive or their absorption is disrupted (Schwiertz et al., 2010). Zhao et al. (2020) indicated that liver lipid synthesis triggered by dietary fructose is dependent on metabolizing fructose to acetic acid and then to Acetyl Coenzyme A through the GM rather than via ATP citrate lyase. The hepatic metabolism of fructose promotes the transcription of genes related to hepatic lipid synthesis, and the metabolite acetate provides the raw material for this process. In addition, SCFAs can induce differentiation of T-cells into Th1 or Th17 cells, depending on the cytokine milieu and the epigenetic activity of histone deacetylases. IL-17 secreted by Th17 cells might play a pro-HCC role by promoting tumor angiogenesis (Liao et al., 2013; Park et al., 2015).

However, emerging evidence suggests the potential protective effect of SCFAs in NAFLD. Sodium butyrate can alleviate HFD-induced intestinal dysbacteriosis and endotoxemia, and thus inhibit NAFLD, by regulating intestinal and liver immune responses (Zhou et al., 2017a). One of the mechanisms is to affect the nutrient intake and metabolism of the host. Butyrate and propionate can activate free fatty acid receptor-3, and thus upregulate the production of the hormones intestine peptide YY and glucagon-like peptide (GLP)-1, which can increase satiety and reduce the intake of energy (Lin et al., 2012). Similarly, activation of GLP-1 has been proven to contribute to the recovery of hepatocyte function, the inhibition of hepatic steatosis and fibrosis, and the prevention of NAFLD developing into NASH (Tang et al., 2015a). SCFAs can also inhibit insulin signal transduction in adipocytes by activating G-protein receptor- 43 , thereby promoting glucose and unbound lipid metabolism, and inhibiting fat accumulation in liver and adipose tissue (Kimura et al., 2013). Another possible mechanism of SCFAs limiting NAFLD is to maintain a healthy intestinal barrier and to weaken inflammatory signals. SCFAs can prevent intestinal mucosal atrophy mediated by GLP-2 (Cani et al., 2009). Increasing the level of GLP-2 through microbial intervention can reduce the intestinal permeability and the levels of LPS and cytokines, thus reducing oxidative stress and liver inflammation (Cani et al., 2009). SCFA supplementation also showed beneficial effects in several inflammatory diseases (e.g., colitis; Rau et al., 2018). By inhibiting colitis, the intestinal barrier can be improved, thereby reducing the liver damage caused by bacterial translocation and the liver transfer of metabolites.

The effects of SCFAs are diverse and extensive, and different kinds and contents of SCFAs in different hosts show different and even contradictory biological effects; therefore, it is difficult to clarify their overall impact (Harris et al., 2020; MartinGallausiaux et al., 2020). In view of the close and complex relationship between SCFAs and host nutrient intake and metabolism, inflammation, and immunity, an in-depth study is needed to determine the specific mechanism by which SCFAs affect the occurrence and development of NAFLD.

\section{Regulation of Abnormal Cholesterol and BA Metabolism Mediated by Diet and the GM}

Lipotoxicity promotes the progression of NASH, fibrosis, cirrhosis, and even HCC (Ioannou, 2016). Among liver lipids, cholesterol is the most important lipotoxic molecule in the development of NAFLD (Ioannou, 2016). Abnormal liver cholesterol homeostasis has been confirmed in both animal models and in humans with NASH. Zhang et al. (2021) revealed the GM-mediated mechanism of dietary cholesterol leading to the progression of NASH, that is, long-term high dietary cholesterol can induce an increase in taurocholic acid and the decrease of 3-indolepropionic acid by changing the GM (decreased levels of Bifidobacterium and Bacteroides and increase levels of Desulfovibrionaceae Anaerotruncus, Desulfovibrio, and Mucispirillum), thus promoting liver lipid accumulation and cell proliferation, leading to the occurrence of NAFLD-HCC (Zhang et al., 2021). In a mouse model, anti-cholesterol treatment eliminated completely the onset of NAFLD-HCC induced by dietary cholesterol (Zhang et al., 2021). This suggests that some of the mechanisms remain unknown: how the related pathogenesis inducing factor (e.g., HFD) causes inflammation, and how to accelerate the transformation of simple hepatic steatosis to NASH, which might be explained by the GM and its metabolites.

BAs are synthesized from cholesterol in the liver and play an important role in the digestion, absorption, and metabolism of fat. The GM is involved in the transformation and metabolism of BAs (Sanchez, 2018). The interaction between BAs and the GM plays an important role in the pathogenesis of NAFLD (Chiang and Ferrell, 2020). BAs participate in the pathogenesis of NAFLD through the farnesoid X receptor (FXR). By binding to FXR, BAs increase insulin sensitivity and reduce hepatic gluconeogenesis and triglyceride in the circulation (Chiang and Ferrell, 2020). Under the intervention of an HFD, the GM promotes weight gain and liver steatosis in an FXR-dependent manner, and the improvement of hepatic steatosis associated with antibiotic therapy depends on FXR signal transduction (Jiang et al., 2015; Parseus et al., 2017). In a large cohort of patients with NASH, although Obecholate (an FXR agonist) did not improve NASH, it significantly improved liver fibrosis compared with that in the control group (Neuschwander-Tetri et al., 2015). BAs also activate Takeda G-protein-coupled receptor 5 (TGR5) in muscle and adipose tissue, thereby increasing energy expenditure (Pols et al., 2011). In addition, activation of TGR5 in the intestine can promote the release of GLP-1, and then positively regulate the secretion of insulin (Pols et al., 2011; Kumar et al., 2016). TGR5 is also expressed in Kupffer cells, which are involved in the regulation of liver inflammation. Activation of TGR5 seems to induce anti-inflammatory effects by inhibiting the NF- $\mathrm{KB}$ signaling pathway and cytokine production (Perino and Schoonjans, 2015). The BA levels in 
liver, serum, and urine were increased in patients with NAFLD (Arab et al., 2017). In a phase II clinical trial in patients with $\mathrm{NASH}$, the BA synthesis inhibitor, Aldafermin, reduced liver inflammation, steatosis, and fibrosis significantly (Harrison et al., 2021). The GM is likely to affect the BA pool, and regulates the metabolism of host cells through the transformation of BAs, including the homeostasis of lipids and glucose; however, its role in the pathogenesis of NAFLD remains controversial, which requires further in-depth study.

\section{Regulation of Choline and Its Derivatives}

Choline deficiency is closely related to the induction and promotion of NAFLD, and is often used to construct animal models of NAFLD (Sherriff et al., 2016). Compared with those in the healthy group, patients with NAFLD generally showed lower levels of serum choline and higher levels of trimethylamine (TMA; Sherriff et al., 2016). In the absence of choline in human body (e.g., because of a choline deficient diet or gut dysbiosis), the synthesis of phosphatidylcholine is insufficient and the level of very-low-density lipoprotein is downregulated, which leads to liver lipid transfer disorder, enhanced mitochondrial $\beta$-oxidative damage, and oxidative stress in hepatocytes, eventually leading to liver steatosis, and aggravating liver inflammation and fibrosis (Smallwood et al., 2016). Some intestinal bacteria (e.g., Desulfovibrio desulfuricans and Escherichia coli) can convert choline to TMA and then to trimethylamine-N-oxide (TMAO) in the liver, which reduces the bioavailability of choline (Sohlenkamp et al., 2003). In addition, TMAO can promote insulin resistance by destroying blood glucose homeostasis and increasing the level of serum inflammatory cytokine C-C motif chemokine ligand 2 (CCL2), and affect lipid metabolism and BA homeostasis by reducing the conversion of cholesterol to BAs, which suggests that TMAO might affect NAFLD indirectly (Tang et al., 2015b). In fact, strategies to reduce TMA and/or TMAO have been used in the clinical treatment or prevention of NAFLD. For example, 3,3-dimethyl-1-butanol, a structural analog of choline, inhibits TMA and TMAO production by inhibiting microbial TMA lyase. However, based on the different microbial characteristics of individuals, it might only be effective for some patients (Wang et al., 2015). Therefore, additional genotyping of the NAFLD cohort is needed to identify patients that would respond to TMA and/or TMAO inhibitors.

\section{Regulation of Other Metabolites}

Endogenous ethanol is produced by some intestinal bacteria via carbohydrate fermentation. Although obese mice with NAFLD did not ingest any alcohol, alcohol could still be detected in their breath (Cope et al., 2000). Compared with healthy individuals or patients with simple hepatic steatosis, the blood ethanol concentration in patients with $\mathrm{NASH}$ is higher, which is associated with increased liver inflammation and liver damage (Baker et al., 2010). Recent studies revealed that about $60 \%$ of patients with NAFLD have high alcohol producing $K$. pneumoniae in their intestines and their abundance is related to the severity of the disease (Yuan et al., 2019). The pathogenesis of NAFLD caused by endogenous ethanol is similar to that of alcoholic fatty liver disease (Parlesak et al., 2000; Seitz et al., 2018; Jennison and Byrne, 2021): (1) the induction of mitochondrial damage and enhanced oxidative stress; (2) destruction of the intestinal barrier and aggravation of liver damage through the gutliver axis; (3) the induction of cytokines, chemokines, Th17, and other immune cells to intensify liver inflammation; and (4) the induction of liver cell damage through acetaldehydemediated cytotoxicity, metabolic disorder, and fat accumulation. These findings not only explain many of the similarities of the pathological features between the two diseases, but also provide a feasible method for clinical diagnosis and treatment of fatty liver caused by such bacteria (Brown and Kleiner, 2016).

Phenylacetic acid (PA) is mainly produced by the metabolism of aromatic amino acids (e.g., phenylalanine) by Bacteroides (Cook, 2019). A multi-omics study showed that PA levels were high in the serum of patients with NASH. At the same time, aromatic amino acids and branched chain amino acids increased, and bacterial diversity decreased (Hoyles et al., 2018). These characteristics have great potential as biomarkers for the clinical diagnosis and prediction of this disease.

Indole, one of the products of tryptophan metabolism by the GM, is generally considered to have anti-inflammatory effects (Yang et al., 2020). Clinical sample analysis, and mouse and cell experiments, showed that indole correlated negatively with NAFLD. Indole supplementation could reduce diet-induced NAFLD, liver fat accumulation, and the inflammatory response in mice. This protective effect was mediated by 6-phosphofructo-2-kinase/fructose-2,6biphosphatase 3 (PFKFB3), a glycolysis regulatory factor of bone marrow cells (Ma et al., 2020). Mimicking or specifically activating PFKFB3 expression in macrophages using indole might be a feasible method to prevent and treat NAFLD and other inflammatory related diseases.

\section{TARGETING THE GM AS A POTENTIAL STRATEGY TO DIAGNOSE AND TREAT NAFLD}

At present, no effective or targeted drug for NAFLD has been approved for marketing. Lifestyle change is still the main intervention for NAFLD; however, the effect and patients' compliance are poor. The close relationship between the GM and NAFLD has been confirmed. Intestinal dysbacteriosis and disturbance of metabolites (type, content, and proportion) and the subsequent metabolic, immune, and inflammatory homeostasis damage might be critical factors for NAFLD development. Thus, targeting the GM is a growing and promising field aiming to slow down and even reverse NAFLD (Sharpton et al., 2021). Moreover, based on the specific changes of the microbiota and metabolites in patients with NAFLD, the GM is also expected to be developed as non-invasive biomarker for the diagnosis, staging, and prognosis of NAFLD (Sharpton et al., 2021). Many studies 
have investigated the feasibility of treating NAFLD by altering the contribution of GM to its pathogenesis, including regulation by fecal microbiota transplantation, probiotics, prebiotics, and synbiotics (Kolodziejczyk et al., 2019). Notably, anti-LPS immunoglobulin, drugs to reverse the BA imbalance in NAFLD (e.g., FXR agonists, peroxisome proliferator activated receptor gamma $(\mathrm{PPAR} \alpha)$ agonists, and ursodeoxycholic acid), and drugs to restore intestinal barrier function and inhibit liver inflammation (e.g., butyrate) have shown encouraging therapeutic effects (Adar et al., 2012; Sun et al., 2018; Yu et al., 2018). Despite the exciting results in many animal studies, the results of multicenter human clinical trials with large samples are still needed. Some representative studies are summarized in Tables 2 and 3.

TABLE 3 | Research on the treatment of NAFLD by targeting GM

\begin{tabular}{|c|c|c|c|c|}
\hline \multicolumn{2}{|c|}{ Intervention factors } & \multirow{2}{*}{$\begin{array}{l}\text { Methods } \\
\text { Clinical trials }\end{array}$} & \multirow{3}{*}{$\begin{array}{l}\text { Treatment results } \\
\text { Hepatic steatosis } \downarrow\end{array}$} & \multirow{3}{*}{$\begin{array}{l}\text { References } \\
\text { Malaguarnera et al., } 2012 \\
\text { Alisi et al., } 2014\end{array}$} \\
\hline \multirow{20}{*}{$\begin{array}{l}\text { Probiotics } \\
\text { (traditional) }\end{array}$} & B. longum & & & \\
\hline & Probiotics VSL\#3 & Clinical trials & & \\
\hline & Lepicol probiotic & Clinical trials & Hepatic triglyceride $\downarrow$, AST $\downarrow$ & Wong et al., 2013b \\
\hline & Parabacteroides distasonis & Pre-clinical trials & $\begin{array}{l}\text { Weight } \downarrow \text {, bile acid, lipid and glucose metabolism } \\
\text { homeostasis } \uparrow\end{array}$ & Wang et al., 2019 \\
\hline & B. xylanisolvens & Pre-clinical trials & $\begin{array}{l}\text { Hepatic butyrate and folate } \uparrow \text {, Fat in liver and } \\
\text { blood } \downarrow\end{array}$ & Qiao et al., 2020 \\
\hline & L. plantarum NCU116 & Pre-clinical trials & ALT $\downarrow$, AST $\downarrow$, lipogenesis $\downarrow$, fatty acid oxidation $\uparrow$ & Li et al., 2014 \\
\hline & L. acidophilus & Clinical trials & $A L T \downarrow, A S T \downarrow$ & Abdel Monem, 2017 \\
\hline & L. acidophilus La5, B. lactis Bb12 & Clinical trials & $\mathrm{ALT} \downarrow, \mathrm{AST} \downarrow, \mathrm{LDL}-\mathrm{C} \downarrow$ & Nabavi et al., 2014 \\
\hline & $\begin{array}{l}\text { L. acidophilus, L. rhamnosus, } \\
\text { B. lactis, B. bifidum }\end{array}$ & Clinical trials & Hepatic steatosis $\downarrow$, TG $\downarrow$, cholesterol $\downarrow$, & Famouri et al., 2017 \\
\hline & $\begin{array}{l}\text { L. acidophilus, L. rhamnosus, } \\
\text { L. paracasei, P. pentosaceus, } \\
\text { B. lactis, B. breve }\end{array}$ & Clinical trials & Total body fat $\downarrow$, TG $\downarrow$, intrahepatic fat $\downarrow$ & Ahn et al., 2019 \\
\hline & L. bulgaricus, S. thermophilus & Clinical trials & ALT $\downarrow, A S T \downarrow$ & Aller et al., 2011 \\
\hline & $\begin{array}{l}\text { L. casei, L. acidophilus, L. } \\
\text { rhamnosus, L. bulgaricus, B. breve, } \\
\text { B. longum, S. thermophilus }\end{array}$ & Clinical trials & Insulin resistance $\downarrow$, TNF- $\alpha \downarrow, \mid L-6 \downarrow$ & Sepideh et al., 2016 \\
\hline & L. johnsonii BS15 & Pre-clinical trials & Hepatic steatosis $\downarrow$, ALT $\downarrow, T G \downarrow$, TNF- $\alpha \downarrow$ & Xin et al., 2014 \\
\hline & L. paracasei & Pre-clinical trials & Hepatic steatosis $\downarrow$, ALT $\downarrow$, TLR4 $\downarrow$, TNF- $\alpha \downarrow$ & Sohn et al., 2015 \\
\hline & L. paracasei N1115 & Pre-clinical trials & Hepatic steatosis $\downarrow$, TNF- $\alpha \downarrow$ & Yao et al., 2019 \\
\hline & L. reuteri GMNL-263 & Pre-clinical trials & Hepatic steatosis $\downarrow$, Liver fibrosis $\downarrow$, TGF- $\beta \downarrow$ & Ting et al., 2015 \\
\hline & L. rhamnosus GG & Pre-clinical trials & Hepatic fat content $\downarrow, T G \downarrow$, cholesterol $\downarrow$ & Kim et al., 2016 \\
\hline & & Clinical trials & TNF- $\alpha \downarrow$, LPS $\downarrow$ & Bajaj et al., 2014 \\
\hline & Protexin & Clinical trials & ALT $\downarrow$, AST $\downarrow$, cholesterol $\downarrow, T G \downarrow$, BMI $\downarrow$ & Shavakhi et al., 2013 \\
\hline & Saccharomyces boulardii & Pre-clinical trials & AST $\downarrow$, endotoxin $\downarrow$, TNF- $\alpha \downarrow$, occludin $\uparrow$ & Liu et al., 2016 \\
\hline \multirow[t]{6}{*}{ Probiotics (novel) } & A. muciniphila & Pre-clinical trials & $\begin{array}{l}\text { Hepatic inflammation } \downarrow \text {, propionate } \uparrow \text {, acetate } \uparrow \text {, } \\
\text { TG } \downarrow \text {, insulin resistance } \downarrow\end{array}$ & $\begin{array}{l}\text { Cani and de Vos, } 2017 \text {; } \\
\text { Moreira et al., } 2018\end{array}$ \\
\hline & Bacteroides spp. & Pre-clinical trials & BMl $\downarrow$, propionate $\uparrow$, acetate $\uparrow, T G \downarrow$ & Tan et al., 2019 \\
\hline & F. prausnitzii & Pre-clinical trials & $\begin{array}{l}\text { Intestinal integrity } \uparrow \text {, hepatic steatosis } \downarrow \text {, hepatic } \\
\text { inflammation } \downarrow\end{array}$ & Munukka et al., 2017 \\
\hline & & Clinical trials & Butyrate $\uparrow$, insulin resistance $\downarrow$ & $\begin{array}{l}\text { Hippe et al., 2016; } \\
\text { Bjorkqvist et al., } 2019\end{array}$ \\
\hline & Roseburia & Clinical trials & Butyrate $\uparrow$, pro-inflammatory cytokines $\downarrow$ & Louis and Flint, 2009 \\
\hline & & Pre-clinical trials & Weight $\downarrow$ & Neyrinck et al., 2012 \\
\hline \multirow[t]{3}{*}{ Prebiotics } & Fructooligosaccharide & Clinical trials & Hepatic steatosis $\downarrow$ & Bomhof et al., 2019 \\
\hline & Inulin & Clinical trials & SCFA $\uparrow$, pro-inflammatory cytokines $\downarrow$ & $\begin{array}{l}\text { Bindels et al., 2012; } \\
\text { Chambers et al., } 2019\end{array}$ \\
\hline & Indole & Pre-clinical trials & Hepatic steatosis $\downarrow$ & Ma et al., 2020 \\
\hline \multirow[t]{3}{*}{ Synbiotics } & Synbiotic 2000Forte & Pre-clinical trials & LPS $\downarrow$ hepatic fibrosis $\downarrow$ & Cortez-Pinto et al., 2016 \\
\hline & L. reuteri and guar gum and inulin & Clinical trials & Hepatic steatosis $\downarrow$, BMI $\downarrow$ & Ferolla et al., 2016 \\
\hline & B. longum and fructooligosaccharide & Clinical trials & $\begin{array}{l}\text { Hepatic steatosis } \downarrow \text {, LPS } \downarrow \text { insulin resistance } \downarrow \text {, } \\
\text { pro-inflammatory cytokines } \downarrow\end{array}$ & Malaguarnera et al., 2012 \\
\hline \multirow[t]{2}{*}{$\begin{array}{l}\text { Fecal microbiota } \\
\text { transplantation }\end{array}$} & Standard diet mice to NASH mice & Pre-clinical trials & $\begin{array}{l}\text { Hepatic steatosis } \downarrow \text {, LPS } \downarrow \text {, butyrate } \uparrow \text {, Intestinal } \\
\text { integrity } \uparrow\end{array}$ & Zhou et al., 2017b \\
\hline & $\begin{array}{l}\text { Healthy and lean donors to NAFLD } \\
\text { acceptor }\end{array}$ & Clinical trials & $\alpha$-Diversity $\uparrow$, butyrate $\uparrow$ insulin resistance $\downarrow$ & Vrieze et al., 2012 \\
\hline
\end{tabular}

L., Lactobacillus; S., Streptococcus; B., Bifidobacterium; P., Pediococcus; ALT, alanine amino transferase; AST, aspartate aminotransferase; LDL-C, low-density lipoprotein cholesterol; TG, triglyceride; TNF, tumor necrosis factor; and BMI, body mass index. 


\section{CONCLUSION AND PROSPECTS}

Along with the lifestyle changes (excessive energy intake and reduced physical activity), NAFLD and its related diseases have become a global epidemic (Younossi, 2019; Huang et al., 2021). It is estimated that the morbidity of NASH will increase by as much as $56 \%$ in the next 10 years, and the incidence of NAFLD-HCC will double by 2030 (Huang et al., 2021). The initiation and progression of NAFLD have been proven to be the liver manifestation of disordered metabolic and immune homeostasis, which may be affected directly or indirectly by GM (Buzzetti et al., 2016; Fang et al., 2018). In recent years, research on the pathogenesis of NAFLD has made breakthroughs; and the advances in GM research have been deepening our understanding of NAFLD, and driving novel diagnostic and therapeutic approaches. However, the complex mechanism of the interaction between the GM and NAFLD has been illusive and limiting clinical progress (Neuschwander-Tetri, 2017; Fang et al., 2018). Whether alterations of the GM and its metabolites are driving factors or a consequence of the development of NAFLD should be further determined in the future.

With the rapid development of next-generation sequencing technology, metagenomics, and non-targeted metabolomics, we have made considerable progress in analyzing the composition and key metabolites of the GM, which has been considered as a potential and valuable non-invasive biomarker to diagnose NAFLD (Ebrahimzadeh Leylabadlo et al., 2020). However, different studies show different and even opposite results (Table 1). In addition, the use of the GM as a biomarker has inherent limitations: it is a highly dynamic aggregate, which is affected, for example, by host genes, living environment, lifestyle, and drugs. Based on massive samples, clinical data, and the results of multi-omics analysis, the combination of dynamic big data and artificial intelligence analysis might produce more reliable information.

The complexity of NAFLD means that there is still no feasible method to reverse the disease process or prevent its occurrence. The clinical significance of specific GMs and metabolite changes associated with NAFLD remains unclear.

\section{REFERENCES}

Abdel Monem, S. M. (2017). Probiotic therapy in patients with nonalcoholic steatohepatitis in Zagazig University hospitals. Euroasian J. Hepatogastroenterol. 7, 101-106. doi: 10.5005/jp-journals-10018-1226

Adar, T., Ben Ya'acov, A., Lalazar, G., Lichtenstein, Y., Nahman, D., Mizrahi, M., et al. (2012). Oral administration of immunoglobulin G-enhanced colostrum alleviates insulin resistance and liver injury and is associated with alterations in natural killer T cells. Clin. Exp. Immunol. 167, 252-260. doi: 10.1111/j. 1365-2249.2011.04511.x

Ahn, S. B., Jun, D. W., Kang, B. K., Lim, J. H., Lim, S., and Chung, M. J. (2019). Randomized, double-blind, placebo-controlled study of a multispecies probiotic mixture in nonalcoholic fatty liver disease. Sci. Rep. 9:5688. doi: 10.1038/s41598-019-42059-3

Albillos, A., De Gottardi, A., and Rescigno, M. (2020). The gut-liver axis in liver disease: pathophysiological basis for therapy. J. Hepatol. 72, 558-577. doi: $10.1016 /$ j.jhep.2019.10.003
The strategy of targeting the GM to reverse the adverse changes of NAFLD has several limitations. Each patient might be associated with different diseases, including obesity and diabetes, and might harbor different predisposing factors, such as genes, diet, and metabolic phenotypes (Wang and Malhi, 2018; Younossi, 2019). A key breakthrough in the future will be the systematic integration of the manifestations, gene expression differences, GM, and metabolic differences in patients with different subtypes of NAFLD. Based on different phenotypes and the application of new technologies to precisely intervene with specific microbiota, it will provide new insights and more accurate treatment for NAFLD. Therefore, using probiotics and prebiotics to fight NAFLD blindly is not recommended until the role of the GM in the pathogenesis of NAFLD is further revealed. It is necessary to understand the functional interactions between the whole microbial community and NAFLD, thus further well-designed clinical trials and evidence-based medical data are needed.

\section{AUTHOR CONTRIBUTIONS}

All authors listed have made a substantial, direct and intellectual contribution to the work, and approved it for publication.

\section{FUNDING}

This work was supported by the National Natural Science Foundation of China (31960236 and 31770536). Lanzhou Chengguan District Science and technology planning project (2020SHFZ0029); Lanzhou talent innovation and Entrepreneurship Project (2019-RC-34); Fund of the first hospital of Lanzhou University (ldyyyn2019-75).

\section{ACKNOWLEDGMENTS}

We would like to thank Jia-qi Yang and Long-fei Ren for providing critical revisions to the manuscript.
Alisi, A., Bedogni, G., Baviera, G., Giorgio, V., Porro, E., Paris, C., et al. (2014). Randomised clinical trial: the beneficial effects of VSL\#3 in obese children with non-alcoholic steatohepatitis. Aliment. Pharmacol. Ther. 39, 1276-1285. doi: 10.1111/apt.12758

Aller, R., De Luis, D. A., Izaola, O., Conde, R., Gonzalez Sagrado, M., Primo, D., et al. (2011). Effect of a probiotic on liver aminotransferases in nonalcoholic fatty liver disease patients: a double blind randomized clinical trial. Eur. Rev. Med. Pharmacol. Sci. 15, 1090-1095.

Arab, J. P., Karpen, S. J., Dawson, P. A., Arrese, M., and Trauner, M. (2017) Bile acids and nonalcoholic fatty liver disease: molecular insights and therapeutic perspectives. Hepatology 65, 350-362. doi: 10.1002/hep.28709

Aron-Wisnewsky, J., Vigliotti, C., Witjes, J., Le, P., Holleboom, A. G., Verheij, J., et al. (2020). Gut microbiota and human NAFLD: disentangling microbial signatures from metabolic disorders. Nat. Rev. Gastroenterol. Hepatol. 17, 279-297. doi: 10.1038/s41575-020-0269-9

Bajaj, J. S., Heuman, D. M., Hylemon, P. B., Sanyal, A. J., Puri, P., Sterling, R. K., et al. (2014). Randomised clinical trial: Lactobacillus GG modulates gut 
microbiome, metabolome and endotoxemia in patients with cirrhosis. Aliment. Pharmacol. Ther. 39, 1113-1125. doi: 10.1111/apt.12695

Baker, S. S., Baker, R. D., Liu, W., Nowak, N. J., and Zhu, L. (2010). Role of alcohol metabolism in non-alcoholic steatohepatitis. PLoS One 5:e9570. doi: 10.1371/journal.pone.0009570

Bindels, L. B., Porporato, P., Dewulf, E. M., Verrax, J., Neyrinck, A. M., Martin, J. C., et al. (2012). Gut microbiota-derived propionate reduces cancer cell proliferation in the liver. Br. J. Cancer 107, 1337-1344. doi: 10.1038/ bjc. 2012.409

Bjorkqvist, O., Repsilber, D., Seifert, M., Brislawn, C., Jansson, J., Engstrand, L., et al. (2019). Alterations in the relative abundance of Faecalibacterium prausnitzii correlate with changes in fecal calprotectin in patients with ileal Crohn's disease: a longitudinal study. Scand. J. Gastroenterol. 54, 577-585. doi: 10.1080/00365521.2019.1599417

Bomhof, M. R., Parnell, J. A., Ramay, H. R., Crotty, P., Rioux, K. P., Probert, C. S., et al. (2019). Histological improvement of non-alcoholic steatohepatitis with a prebiotic: a pilot clinical trial. Eur. J. Nutr. 58, 1735-1745. doi: 10.1007/ s00394-018-1721-2

Boursier, J., Mueller, O., Barret, M., Machado, M., Fizanne, L., Araujo-Perez, F., et al. (2016). The severity of nonalcoholic fatty liver disease is associated with gut dysbiosis and shift in the metabolic function of the gut microbiota. Hepatology 63, 764-775. doi: 10.1002/hep.28356

Brown, G. T., and Kleiner, D. E. (2016). Histopathology of nonalcoholic fatty liver disease and nonalcoholic steatohepatitis. Metabolism 65, 1080-1086. doi: 10.1016/j.metabol.2015.11.008

Brunt, E. M., Wong, V. W., Nobili, V., Day, C. P., Sookoian, S., Maher, J. J., et al. (2015). Nonalcoholic fatty liver disease. Nat. Rev. Dis. Primers 1:15080. doi: $10.1038 / \mathrm{nrdp} .2015 .80$

Buzzetti, E., Pinzani, M., and Tsochatzis, E. A. (2016). The multiple-hit pathogenesis of non-alcoholic fatty liver disease (NAFLD). Metabolism 65, 1038-1048. doi: 10.1016/j.metabol.2015.12.012

Canfora, E. E., Meex, R. C. R., Venema, K., and Blaak, E. E. (2019). Gut microbial metabolites in obesity, NAFLD and T2DM. Nat. Rev. Endocrinol. 15, 261-273. doi: 10.1038/s41574-019-0156-Z

Cani, P. D., and De Vos, W. M. (2017). Next-generation beneficial microbes: the case of Akkermansia muciniphila. Front. Microbiol. 8:1765. doi: 10.3389/ fmicb.2017.01765

Cani, P. D., Possemiers, S., Van De Wiele, T., Guiot, Y., Everard, A., Rottier, O., et al. (2009). Changes in gut microbiota control inflammation in obese mice through a mechanism involving GLP-2-driven improvement of gut permeability. Gut 58, 1091-1103. doi: 10.1136/gut.2008.165886

Cano, P. G., Santacruz, A., Trejo, F. M., and Sanz, Y. (2013). Bifidobacterium CECT 7765 improves metabolic and immunological alterations associated with obesity in high-fat diet-fed mice. Obesity 21, 2310-2321. doi: 10.1002/ oby. 20330

Carpino, G., Del Ben, M., Pastori, D., Carnevale, R., Baratta, F., Overi, D., et al. (2020). Increased liver localization of lipopolysaccharides in human and experimental NAFLD. Hepatology 72, 470-485. doi: 10.1002/hep.31056

Caussy, C., Hsu, C., Lo, M. T., Liu, A., Bettencourt, R., Ajmera, V. H., et al. (2018). Link between gut-microbiome derived metabolite and shared geneeffects with hepatic steatosis and fibrosis in NAFLD. Hepatology 68, 918-932. doi: 10.1002/hep. 29892

Chambers, E. S., Byrne, C. S., Rugyendo, A., Morrison, D. J., Preston, T., Tedford, C., et al. (2019). The effects of dietary supplementation with inulin and inulin-propionate ester on hepatic steatosis in adults with non-alcoholic fatty liver disease. Diabetes Obes. Metab. 21, 372-376. doi: 10.1111/dom. 13500

Chen, F., Esmaili, S., Rogers, G. B., Bugianesi, E., Petta, S., Marchesini, G., et al. (2020). Lean NAFLD: a distinct entity shaped by differential metabolic adaptation. Hepatology 71, 1213-1227. doi: 10.1002/hep.30908

Chiang, J. Y. L., and Ferrell, J. M. (2020). Bile acid receptors FXR and TGR5 signaling in fatty liver diseases and therapy. Am. J. Physiol. Gastrointest. Liver Physiol. 318, G554-G573. doi: 10.1152/ajpgi.00223.2019

Chu, H. K., Duan, Y., Yang, L., and Schnabl, B. (2019). Small metabolites, possible big changes: a microbiota-centered view of non-alcoholic fatty liver disease. Gut 68, 359-370. doi: 10.1136/gutjnl-2018-316307

Cook, S. D. (2019). An historical review of phenylacetic acid. Plant Cell Physiol. 60, 243-254. doi: 10.1093/pcp/pcz004
Cope, K., Risby, T., and Diehl, A. M. (2000). Increased gastrointestinal ethanol production in obese mice: implications for fatty liver disease pathogenesis. Gastroenterology 119, 1340-1347. doi: 10.1053/gast.2000.19267

Cortez-Pinto, H., Borralho, P., Machado, J., Lopes, M. T., Gato, I. V., Santos, A. M., et al. (2016). Microbiota modulation with synbiotic decreases liver fibrosis in a high fat choline deficient diet mice model of non-alcoholic Steatohepatitis (NASH). GE Port. J. Gastroenterol. 23, 132-141. doi: 10.1016/j.jpge.2016.01.004

Da Silva, H. E., Teterina, A., Comelli, E. M., Taibi, A., Arendt, B. M., Fischer, S. E., et al. (2018). Nonalcoholic fatty liver disease is associated with dysbiosis independent of body mass index and insulin resistance. Sci. Rep. 8:1466. doi: 10.1038/s41598-018-19753-9

De Santis, S., Cavalcanti, E., Mastronardi, M., Jirillo, E., and Chieppa, M. (2015). Nutritional keys for intestinal barrier modulation. Front. Immunol. 6:612. doi: 10.3389/fimmu.2015.00612

Del Chierico, F., Nobili, V., Vernocchi, P., Russo, A., De Stefanis, C., Gnani, D., et al. (2017). Gut microbiota profiling of pediatric nonalcoholic fatty liver disease and obese patients unveiled by an integrated meta-omics-based approach. Hepatology 65, 451-464. doi: 10.1002/hep.28572

Ebrahimzadeh Leylabadlo, H., Ghotaslou, R., Samadi Kafil, H., Feizabadi, M. M., Moaddab, S. Y., Farajnia, S., et al. (2020). Nonalcoholic fatty liver diseases: from role of gut microbiota to microbialbased therapies. Eur. J. Clin. Microbiol. Infect. Dis. 39, 613-627. doi: 10.1007/s10096-019-03746-1

Ezzaidi, N., Zhang, X., Coker, O. O., and Yu, J. (2019). New insights and therapeutic implication of gut microbiota in non-alcoholic fatty liver disease and its associated liver cancer. Cancer Lett. 459, 186-191. doi: 10.1016/j. canlet.2019.114425

Famouri, F., Shariat, Z., Hashemipour, M., Keikha, M., and Kelishadi, R. (2017). Effects of probiotics on nonalcoholic fatty liver disease in obese children and adolescents. J. Pediatr. Gastroenterol. Nutr. 64, 413-417. doi: 10.1097/ MPG.0000000000001422

Fang, Y. L., Chen, H., Wang, C. L., and Liang, L. (2018). Pathogenesis of non-alcoholic fatty liver disease in children and adolescence: from "two hit theory" to "multiple hit model". World J. Gastroenterol. 24, 2974-2983. doi: 10.3748/wjg.v24.i27.2974

Ferolla, S. M., Couto, C. A., Costa-Silva, L., Armiliato, G. N., Pereira, C. A., Martins, F. S., et al. (2016). Beneficial effect of synbiotic supplementation on hepatic steatosis and anthropometric parameters, but not on gut permeability in a population with nonalcoholic steatohepatitis. Nutrients 8:397. doi: 10.3390/ nu8070397

Gabele, E., Dostert, K., Hofmann, C., Wiest, R., Scholmerich, J., Hellerbrand, C., et al. (2011). DSS induced colitis increases portal LPS levels and enhances hepatic inflammation and fibrogenesis in experimental NASH. J. Hepatol. 55, 1391-1399. doi: 10.1016/j.jhep.2011.02.035

Gomes, A. C., Hoffmann, C., and Mota, J. F. (2018). The human gut microbiota: metabolism and perspective in obesity. Gut Microbes 9, 308-325. doi: 10.1080/19490976.2018.1465157

Gong, S., Lan, T., Zeng, L., Luo, H., Yang, X., Li, N., et al. (2018). Gut microbiota mediates diurnal variation of acetaminophen induced acute liver injury in mice. J. Hepatol. 69, 51-59. doi: 10.1016/j.jhep.2018.02.024

Hand, T. W., Vujkovic-Cvijin, I., Ridaura, V. K., and Belkaid, Y. (2016). Linking the microbiota, chronic disease, and the immune system. Trends Endocrinol. Metab. 27, 831-843. doi: 10.1016/j.tem.2016.08.003

Harris, H. C., Morrison, D. J., and Edwards, C. A. (2020). Impact of the source of fermentable carbohydrate on SCFA production by human gut microbiota in vitro: a systematic scoping review and secondary analysis. Crit. Rev. Food Sci. Nutr., 1-12. doi: 10.1080/10408398.2020.1809991

Harrison, S. A., Neff, G., Guy, C. D., Bashir, M. R., Paredes, A. H., Frias, J. P., et al. (2021). Efficacy and safety of aldafermin, an engineered FGF19 analog, in a randomized, double-blind, placebo-controlled trial of patients with nonalcoholic steatohepatitis. Gastroenterology 160, 219.e1-231.e1. doi: 10.1053/j. gastro.2020.08.004

He, L. H., Ren, L. F., Li, J. F., Wu, Y. N., Li, X., and Zhang, L. (2020). Intestinal flora as a potential strategy to fight SARS-CoV-2 infection. Front. Microbiol. 11:1388. doi: 10.3389/fmicb.2020.01388

Henao-Mejia, J., Elinav, E., Jin, C., Hao, L., Mehal, W. Z., Strowig, T., et al. (2012). Inflammasome-mediated dysbiosis regulates progression of NAFLD and obesity. Nature 482, 179-185. doi: 10.1038/nature10809 
Hippe, B., Remely, M., Aumueller, E., Pointner, A., Magnet, U., and Haslberger, A. G. (2016). Faecalibacterium prausnitzii phylotypes in type two diabetic, obese, and lean control subjects. Benefic. Microbes 7, 511-517. doi: 10.3920/ BM2015.0075

Hoyles, L., Fernandez-Real, J. M., Federici, M., Serino, M., Abbott, J., Charpentier, J., et al. (2018). Molecular phenomics and metagenomics of hepatic steatosis in non-diabetic obese women. Nat. Med. 24, 1070-1080. doi: 10.1038/ s41591-018-0061-3

Hu, H., Lin, A., Kong, M., Yao, X., Yin, M., Xia, H., et al. (2020). Intestinal microbiome and NAFLD: molecular insights and therapeutic perspectives. J. Gastroenterol. 55, 142-158. doi: 10.1007/s00535-019-01649-8

Huang, D. Q., El-Serag, H. B., and Loomba, R. (2021). Global epidemiology of NAFLD-related HCC: trends, predictions, risk factors and prevention. Nat. Rev. Gastroenterol. Hepatol. 18, 223-238. doi: 10.1038/s41575020-00381-6

Ioannou, G. N. (2016). The role of cholesterol in the pathogenesis of NASH. Trends Endocrinol. Metab. 27, 84-95. doi: 10.1016/j.tem.2015.11.008

Ipsen, D. H., Lykkesfeldt, J., and Tveden-Nyborg, P. (2018). Molecular mechanisms of hepatic lipid accumulation in non-alcoholic fatty liver disease. Cell. Mol. Life Sci. 75, 3313-3327. doi: 10.1007/s00018-018-2860-6

Jennison, E., and Byrne, C. D. (2021). The role of the gut microbiome and diet in the pathogenesis of non-alcoholic fatty liver disease. Clin. Mol. Hepatol. 27, 22-43. doi: 10.3350/cmh.2020.0129

Jiang, C., Xie, C., Li, F., Zhang, L., Nichols, R. G., Krausz, K. W., et al. (2015). Intestinal farnesoid $\mathrm{X}$ receptor signaling promotes nonalcoholic fatty liver disease. J. Clin. Invest. 125, 386-402. doi: 10.1172/JCI76738

Kim, H. N., Joo, E. J., Cheong, H. S., Kim, Y., Kim, H. L., Shin, H., et al. (2019). Gut microbiota and risk of persistent nonalcoholic fatty liver diseases. J. Clin. Med. 8:1089. doi: 10.3390/jcm8081089

Kim, B., Park, K. Y., Ji, Y., Park, S., Holzapfel, W., and Hyun, C. K. (2016). Protective effects of Lactobacillus rhamnosus GG against dyslipidemia in high-fat diet-induced obese mice. Biochem. Biophys. Res. Commun. 473, 530-536. doi: 10.1016/j.bbrc.2016.03.107

Kimura, I., Ozawa, K., Inoue, D., Imamura, T., Kimura, K., Maeda, T., et al. (2013). The gut microbiota suppresses insulin-mediated fat accumulation via the short-chain fatty acid receptor GPR43. Nat. Commun. 4:1829. doi: 10.1038/ncomms 2852

Kolodziejczyk, A. A., Zheng, D., Shibolet, O., and Elinav, E. (2019). The role of the microbiome in NAFLD and NASH. EMBO Mol. Med. 11:e9302. doi: 10.15252/emmm.201809302

Kumar, D. P., Asgharpour, A., Mirshahi, F., Park, S. H., Liu, S., Imai, Y., et al. (2016). Activation of transmembrane bile acid receptor TGR5 modulates pancreatic islet alpha cells to promote glucose homeostasis. J. Biol. Chem. 291, 6626-6640. doi: 10.1074/jbc.M115.699504

Le Roy, T., Llopis, M., Lepage, P., Bruneau, A., Rabot, S., Bevilacqua, C., et al. (2013). Intestinal microbiota determines development of non-alcoholic fatty liver disease in mice. Gut 62, 1787-1794. doi: 10.1136/gutjnl2012-303816

Lelouvier, B., Servant, F., Paisse, S., Brunet, A. C., Benyahya, S., Serino, M., et al. (2016). Changes in blood microbiota profiles associated with liver fibrosis in obese patients: a pilot analysis. Hepatology 64, 2015-2027. doi: 10.1002/hep.28829

Li, C., Nie, S. P., Zhu, K. X., Ding, Q., Li, C., Xiong, T., et al. (2014). Lactobacillus plantarum NCU116 improves liver function, oxidative stress and lipid metabolism in rats with high fat diet induced non-alcoholic fatty liver disease. Food Funct. 5, 3216-3223. doi: 10.1039/C4FO00549J

Li, F., Sun, G., Wang, Z., Wu, W., Guo, H., Peng, L., et al. (2018). Characteristics of fecal microbiota in non-alcoholic fatty liver disease patients. Sci. China Life Sci. 61, 770-778. doi: 10.1007/s11427-017-9303-9

Liao, R., Sun, J., Wu, H., Yi, Y., Wang, J. X., He, H. W., et al. (2013). High expression of IL-17 and IL-17RE associate with poor prognosis of hepatocellular carcinoma. J. Exp. Clin. Cancer Res. 32:3. doi: 10.1186/1756-9966-32-3

Lin, H. V., Frassetto, A., Kowalik, E. J. Jr., Nawrocki, A. R., Lu, M. M., Kosinski, J. R., et al. (2012). Butyrate and propionate protect against dietinduced obesity and regulate gut hormones via free fatty acid receptor 3-independent mechanisms. PLoS One 7:e35240. doi: 10.1371/journal. pone.0035240

Liu, Y. T., Li, Y. Q., and Wang, Y. Z. (2016). Protective effect of Saccharomyces boulardii against intestinal mucosal barrier injury in rats with nonalcoholic fatty liver disease. Zhonghua Gan Zang Bing Za Zhi 24, 921-926. doi: 10.3760/cma.j.issn.1007-3418.2016.12.009

Loomba, R., Seguritan, V., Li, W., Long, T., Klitgord, N., Bhatt, A., et al. (2017). Gut microbiome-based metagenomic signature for non-invasive detection of advanced fibrosis in human nonalcoholic fatty liver disease. Cell Metab. 25, 1054.e1055-1062.e1055. doi: 10.1016/j.cmet.2017.04.001

Loomba, R., Seguritan, V., Li, W., Long, T., Klitgord, N., Bhatt, A., et al. (2019). Gut microbiome-based metagenomic signature for non-invasive detection of advanced fibrosis in human nonalcoholic fatty liver disease. Cell Metab. 30:607. doi: 10.1016/j.cmet.2019.08.002

Louis, P., and Flint, H. J. (2009). Diversity, metabolism and microbial ecology of butyrate-producing bacteria from the human large intestine. FEMS Microbiol. Lett. 294, 1-8. doi: 10.1111/j.1574-6968.2009.01514.x

Luther, J., Garber, J. J., Khalili, H., Dave, M., Bale, S. S., Jindal, R., et al. (2015). Hepatic injury in nonalcoholic steatohepatitis contributes to altered intestinal permeability. Cell. Mol. Gastroenterol. Hepatol. 1, 222-232. doi: 10.1016/j.jcmgh.2015.01.001

Ma, L., Li, H., Hu, J., Zheng, J., Zhou, J., Botchlett, R., et al. (2020). Indole alleviates diet-induced hepatic steatosis and inflammation in a manner involving myeloid cell 6-phosphofructo-2-kinase/fructose-2,6-biphosphatase 3. Hepatology 72, 1191-1203. doi: 10.1002/hep.31115

Malaguarnera, M., Vacante, M., Antic, T., Giordano, M., Chisari, G., Acquaviva, R., et al. (2012). Bifidobacterium longum with fructo-oligosaccharides in patients with non alcoholic steatohepatitis. Dig. Dis. Sci. 57, 545-553. doi: 10.1007/ s10620-011-1887-4

Martens, E. C., Neumann, M., and Desai, M. S. (2018). Interactions of commensal and pathogenic microorganisms with the intestinal mucosal barrier. Nat. Rev. Microbiol. 16, 457-470. doi: 10.1038/s41579-018-0036-x

Martin-Gallausiaux, C., Marinelli, L., Blottiere, H. M., Larraufie, P., and Lapaque, N. (2020). SCFA: mechanisms and functional importance in the gut. Proc. Nutr. Soc. 80, 37-49. doi: 10.1017/S0029665120006916

Michail, S., Lin, M., Frey, M. R., Fanter, R., Paliy, O., Hilbush, B., et al. (2015). Altered gut microbial energy and metabolism in children with non-alcoholic fatty liver disease. FEMS Microbiol. Ecol. 91, 1-9. doi: 10.1093/ femsec/fiu002

Moreira, G. V., Azevedo, F. F., Ribeiro, L. M., Santos, A., Guadagnini, D., Gama, P., et al. (2018). Liraglutide modulates gut microbiota and reduces NAFLD in obese mice. J. Nutr. Biochem. 62, 143-154. doi: 10.1016/j. jnutbio.2018.07.009

Morrison, D. J., and Preston, T. (2016). Formation of short chain fatty acids by the gut microbiota and their impact on human metabolism. Gut Microbes 7, 189-200. doi: 10.1080/19490976.2015.1134082

Mouries, J., Brescia, P., Silvestri, A., Spadoni, I., Sorribas, M., Wiest, R., et al. (2019). Microbiota-driven gut vascular barrier disruption is a prerequisite for non-alcoholic steatohepatitis development. J. Hepatol. 71, 1216-1228. doi: 10.1016/j.jhep.2019.08.005

Mouzaki, M., Comelli, E. M., Arendt, B. M., Bonengel, J., Fung, S. K., Fischer, S. E., et al. (2013). Intestinal microbiota in patients with nonalcoholic fatty liver disease. Hepatology 58, 120-127. doi: 10.1002/hep.26319

Mridha, A. R., Wree, A., Robertson, A. A. B., Yeh, M. M., Johnson, C. D., Van Rooyen, D. M., et al. (2017). NLRP3 inflammasome blockade reduces liver inflammation and fibrosis in experimental NASH in mice. J. Hepatol. 66, 1037-1046. doi: 10.1016/j.jhep.2017.01.022

Munukka, E., Rintala, A., Toivonen, R., Nylund, M., Yang, B., Takanen, A., et al. (2017). Faecalibacterium prausnitzii treatment improves hepatic health and reduces adipose tissue inflammation in high-fat fed mice. ISME J. 11, 1667-1679. doi: 10.1038/ismej.2017.24

Nabavi, S., Rafraf, M., Somi, M. H., Homayouni-Rad, A., and Asghari-Jafarabadi, M. (2014). Effects of probiotic yogurt consumption on metabolic factors in individuals with nonalcoholic fatty liver disease. J. Dairy Sci. 97, 7386-7393. doi: $10.3168 /$ jds.2014-8500

Neuschwander-Tetri, B. A. (2017). Non-alcoholic fatty liver disease. BMC Med. 15:45. doi: 10.1186/s12916-017-0806-8

Neuschwander-Tetri, B. A., Loomba, R., Sanyal, A. J., Lavine, J. E., Van Natta, M. L., Abdelmalek, M. F., et al. (2015). Farnesoid X nuclear receptor ligand obeticholic acid for non-cirrhotic, non-alcoholic steatohepatitis (FLINT): a multicentre, randomised, placebo-controlled trial. Lancet 385, 956-965. doi: 10.1016/S0140-6736(14)61933-4 
Neyrinck, A. M., Possemiers, S., Verstraete, W., De Backer, F., Cani, P. D., and Delzenne, N. M. (2012). Dietary modulation of clostridial cluster XIVa gut bacteria (Roseburia spp.) by chitin-glucan fiber improves host metabolic alterations induced by high-fat diet in mice. J. Nutr. Biochem. 23, 51-59. doi: 10.1016/j.jnutbio.2010.10.008

Ozkul, C., Yalinay, M., Karakan, T., and Yilmaz, G. (2017). Determination of certain bacterial groups in gut microbiota and endotoxin levels in patients with nonalcoholic steatohepatitis. Turk J Gastroenterol 28, 361-369. doi: 10.5152/tjg.2017.17033

Park, J., Kim, M., Kang, S. G., Jannasch, A. H., Cooper, B., Patterson, J., et al. (2015). Short-chain fatty acids induce both effector and regulatory $\mathrm{T}$ cells by suppression of histone deacetylases and regulation of the mTOR-S6K pathway. Mucosal Immunol. 8, 80-93. doi: 10.1038/mi.2014.44

Parlesak, A., Schafer, C., Schutz, T., Bode, J. C., and Bode, C. (2000). Increased intestinal permeability to macromolecules and endotoxemia in patients with chronic alcohol abuse in different stages of alcohol-induced liver disease. J. Hepatol. 32, 742-747. doi: 10.1016/s0168-8278(00)80242-1

Parseus, A., Sommer, N., Sommer, F., Caesar, R., Molinaro, A., Stahlman, M., et al. (2017). Microbiota-induced obesity requires farnesoid X receptor. Gut 66, 429-437. doi: 10.1136/gutjnl-2015-310283

Perino, A., and Schoonjans, K. (2015). TGR5 and immunometabolism: insights from physiology and pharmacology. Trends Pharmacol. Sci. 36, 847-857. doi: 10.1016/j.tips.2015.08.002

Peterson, L. W., and Artis, D. (2014). Intestinal epithelial cells: regulators of barrier function and immune homeostasis. Nat. Rev. Immunol. 14, 141-153. doi: $10.1038 /$ nri3608

Plociennikowska, A., Hromada-Judycka, A., Borzecka, K., and Kwiatkowska, K. (2015). Co-operation of TLR4 and raft proteins in LPS-induced proinflammatory signaling. Cell. Mol. Life Sci. 72, 557-581. doi: 10.1007/ s00018-014-1762-5

Pols, T. W. H., Noriega, L. G., Nomura, M., Auwerx, J., and Schoonjans, K. (2011). The bile acid membrane receptor TGR5 as an emerging target in metabolism and inflammation. J. Hepatol. 54, 1263-1272. doi: 10.1016/j. jhep.2010.12.004

Qiao, S., Bao, L., Wang, K., Sun, S., Liao, M., Liu, C., et al. (2020). Activation of a specific gut bacteroides-folate-liver axis benefits for the alleviation of nonalcoholic hepatic steatosis. Cell Rep. 32:108005. doi: 10.1016/j. celrep.2020.108005

Rahman, K., Desai, C., Iyer, S. S., Thorn, N. E., Kumar, P., Liu, Y., et al. (2016). Loss of junctional adhesion molecule a promotes severe steatohepatitis in mice on a diet high in saturated fat, fructose, and cholesterol. Gastroenterology 151, 733.e712-746.e712. doi: 10.1053/j.gastro.2016.06.022

Raman, M., Ahmed, I., Gillevet, P. M., Probert, C. S., Ratcliffe, N. M., Smith, S., et al. (2013). Fecal microbiome and volatile organic compound metabolome in obese humans with nonalcoholic fatty liver disease. Clin. Gastroenterol. Hepatol. 11, 868.e861-863-875.e861-863. doi: 10.1016/j.cgh.2013.02.015

Rau, M., Rehman, A., Dittrich, M., Groen, A. K., Hermanns, H. M., Seyfried, F., et al. (2018). Fecal SCFAs and SCFA-producing bacteria in gut microbiome of human NAFLD as a putative link to systemic T-cell activation and advanced disease. United European Gastroenterol J 6, 1496-1507. doi: 10.1177/2050640618804444

Sanchez, B. (2018). Bile acid-microbiota crosstalk in gastrointestinal inflammation and carcinogenesis: a role for bifidobacteria and lactobacilli? Nat. Rev. Gastroenterol. Hepatol. 15:205. doi: 10.1038/nrgastro.2018.23

Schirmer, M., Franzosa, E. A., Lloyd-Price, J., Mciver, L. J., Schwager, R., Poon, T. W., et al. (2018). Dynamics of metatranscription in the inflammatory bowel disease gut microbiome. Nat. Microbiol. 3, 337-346. doi: 10.1038/ s41564-017-0089-z

Schwiertz, A., Taras, D., Schafer, K., Beijer, S., Bos, N. A., Donus, C., et al. (2010). Microbiota and SCFA in lean and overweight healthy subjects. Obesity 18, 190-195. doi: 10.1038/oby.2009.167

Schwimmer, J. B., Johnson, J. S., Angeles, J. E., Behling, C., Belt, P. H., Borecki, I., et al. (2019). Microbiome signatures associated with steatohepatitis and moderate to severe fibrosis in children with nonalcoholic fatty liver disease. Gastroenterology 157, 1109-1122. doi: 10.1053/j.gastro.2019.06.028

Seitz, H. K., Bataller, R., Cortez-Pinto, H., Gao, B., Gual, A., Lackner, C., et al. (2018). Alcoholic liver disease. Nat. Rev. Dis. Primers 4:16. doi: 10.1038/ s41572-018-0021-8
Sender, R., Fuchs, S., and Milo, R. (2016). Are we really vastly outnumbered? Revisiting the ratio of bacterial to host cells in humans. Cell 164, 337-340. doi: 10.1016/j.cell.2016.01.013

Sepideh, A., Karim, P., Hossein, A., Leila, R., Hamdollah, M., Mohammad, E. G., et al. (2016). Effects of multistrain probiotic supplementation on glycemic and inflammatory indices in patients with nonalcoholic fatty liver disease: a double-blind randomized clinical trial. J. Am. Coll. Nutr. 35, 500-505. doi: 10.1080/07315724.2015.1031355

Sharpton, S. R., Schnabl, B., Knight, R., and Loomba, R. (2021). Current concepts, opportunities, and challenges of gut microbiome-based personalized medicine in nonalcoholic fatty liver disease. Cell Metab. 33, 21-32. doi: 10.1016/j.cmet.2020.11.010

Shavakhi, A., Minakari, M., Firouzian, H., Assali, R., Hekmatdoost, A., and Ferns, G. (2013). Effect of a probiotic and metformin on liver aminotransferases in non-alcoholic steatohepatitis: a double blind randomized clinical trial. Int. J. Prev. Med. 4, 531-537.

Shen, F., Zheng, R. D., Sun, X. Q., Ding, W. J., Wang, X. Y., and Fan, J. G. (2017). Gut microbiota dysbiosis in patients with non-alcoholic fatty liver disease. Hepatobiliary Pancreat. Dis. Int. 16, 375-381. doi: 10.1016/ S1499-3872(17)60019-5

Sherriff, J. L., O'sullivan, T. A., Properzi, C., Oddo, J. L., and Adams, L. A. (2016). Choline, its potential role in nonalcoholic fatty liver disease, and the case for human and bacterial genes. Adv. Nutr. 7, 5-13. doi: 10.3945/an.114.007955

Smallwood, T., Allayee, H., and Bennett, B. J. (2016). Choline metabolites: gene by diet interactions. Curr. Opin. Lipidol. 27, 33-39. doi: 10.1097/ MOL.0000000000000259

Sobhonslidsuk, A., Chanprasertyothin, S., Pongrujikorn, T., Kaewduang, P., Promson, K., Petraksa, S., et al. (2018). The association of gut microbiota with nonalcoholic steatohepatitis in thais. Biomed. Res. Int. 2018:9340316. doi: $10.1155 / 2018 / 9340316$

Sohlenkamp, C., Lopez-Lara, I. M., and Geiger, O. (2003). Biosynthesis of phosphatidylcholine in bacteria. Prog. Lipid Res. 42, 115-162. doi: 10.1016/ S0163-7827(02)00050-4

Sohn, W., Jun, D. W., Lee, K. N., Lee, H. L., Lee, O. Y., Choi, H. S., et al. (2015). Lactobacillus paracasei induces M2-dominant kupffer cell polarization in a mouse model of nonalcoholic steatohepatitis. Dig. Dis. Sci. 60, 3340-3350. doi: 10.1007/s10620-015-3770-1

Spencer, M. D., Hamp, T. J., Reid, R. W., Fischer, L. M., Zeisel, S. H., and Fodor, A. A. (2011). Association between composition of the human gastrointestinal microbiome and development of fatty liver with choline deficiency. Gastroenterology 140, 976-986. doi: 10.1053/j.gastro.2010.11.049

Sun, B., Jia, Y., Hong, J., Sun, Q., Gao, S., Hu, Y., et al. (2018). Sodium butyrate ameliorates high-fat-diet-induced non-alcoholic fatty liver disease through peroxisome proliferator-activated receptor alpha-mediated activation of beta oxidation and suppression of inflammation. J. Agric. Food Chem. 66, 7633-7642. doi: 10.1021/acs.jafc.8b01189

Szabo, G. (2015). Gut-liver axis in alcoholic liver disease. Gastroenterology 148, 30-36. doi: 10.1053/j.gastro.2014.10.042

Tan, H., Zhai, Q., and Chen, W. (2019). Investigations of Bacteroides spp. towards next-generation probiotics. Food Res. Int. 116, 637-644. doi: 10.1016/j. foodres.2018.08.088

Tang, A., Rabasa-Lhoret, R., Castel, H., Wartelle-Bladou, C., Gilbert, G., Massicotte-Tisluck, K., et al. (2015a). Effects of insulin glargine and liraglutide therapy on liver fat as measured by magnetic resonance in patients with type 2 diabetes: a randomized trial. Diabetes Care 38, 1339-1346. doi: $10.2337 / \mathrm{dc} 14-2548$

Tang, W. H. W., Wang, Z. N., Kennedy, D. J., Wu, Y. P., Buffa, J. A., Agatisa-Boyle, B., et al. (2015b). Gut microbiota-dependent trimethylamine N-oxide (TMAO) pathway contributes to both development of renal insufficiency and mortality risk in chronic kidney disease. Circ. Res. 116, 448-455. doi: 10.1161/ Circresaha.116.305360

Ting, W. J., Kuo, W. W., Hsieh, D. J., Yeh, Y. L., Day, C. H., Chen, Y. H., et al. (2015). Heat killed Lactobacillus reuteri GMNL-263 reduces fibrosis effects on the liver and heart in high fat diet-hamsters via TGF-beta suppression. Int. J. Mol. Sci. 16, 25881-25896. doi: 10.3390/ijms161025881

Tripathi, A., Debelius, J., Brenner, D. A., Karin, M., Loomba, R., Schnabl, B., et al. (2018). The gut-liver axis and the intersection with the microbiome. Nat. Rev. Gastroenterol. Hepatol. 15, 397-411. doi: 10.1038/s41575-018-0011-z 
Tsai, M. C., Liu, Y. Y., Lin, C. C., Wang, C. C., Wu, Y. J., Yong, C. C., et al. (2020). Gut microbiota dysbiosis in patients with biopsy-proven nonalcoholic fatty liver disease: a cross-sectional study in Taiwan. Nutrients 12:820. doi: 10.3390/nu12030820

Vrieze, A., Van Nood, E., Holleman, F., Salojarvi, J., Kootte, R. S., Bartelsman, J. F., et al. (2012). Transfer of intestinal microbiota from lean donors increases insulin sensitivity in individuals with metabolic syndrome. Gastroenterology 143, 913.e917-916.e917. doi: 10.1053/j.gastro.2012.06.031

Wang, B., Jiang, X., Cao, M., Ge, J., Bao, Q., Tang, L., et al. (2016). Altered fecal microbiota correlates with liver biochemistry in nonobese patients with non-alcoholic fatty liver disease. Sci. Rep. 6:32002. doi: 10.1038/srep32002

Wang, K., Liao, M., Zhou, N., Bao, L., Ma, K., Zheng, Z., et al. (2019). Parabacteroides distasonis alleviates obesity and metabolic dysfunctions via production of succinate and secondary bile acids. Cell Rep. 26, 222. e225-235.e225. doi: 10.1016/j.celrep.2018.12.028

Wang, X. J., and Malhi, H. (2018). Nonalcoholic fatty liver disease. Ann. Intern. Med. 169, ITC65-ITC80. doi: 10.7326/AITC201811060

Wang, Z. N., Roberts, A. B., Buffa, J. A., Levison, B. S., Zhu, W. F., Org, E., et al. (2015). Non-lethal inhibition of gut microbial trimethylamine production for the treatment of atherosclerosis. Cell 163, 1585-1595. doi: 10.1016/j.cell.2015.11.055

Wong, V. W., Tse, C. H., Lam, T. T., Wong, G. L., Chim, A. M., Chu, W. C., et al. (2013a). Molecular characterization of the fecal microbiota in patients with nonalcoholic steatohepatitis: a longitudinal study. PLoS One 8:e62885. doi: 10.1371/journal.pone.0062885

Wong, V. W., Won, G. L., Chim, A. M., Chu, W. C., Yeung, D. K., Li, K. C., et al. (2013b). Treatment of nonalcoholic steatohepatitis with probiotics. A proof-of-concept study. Ann. Hepatol. 12, 256-262.

Xin, J., Zeng, D., Wang, H., Ni, X., Yi, D., Pan, K., et al. (2014). Preventing non-alcoholic fatty liver disease through lactobacillus johnsonii BS15 by attenuating inflammation and mitochondrial injury and improving gut environment in obese mice. Appl. Microbiol. Biotechnol. 98, 6817-6829. doi: 10.1007/s00253-014-5752-1

Yang, J., Chawla, R., Rhee, K. Y., Gupta, R., Manson, M. D., Jayaraman, A., et al. (2020). Biphasic chemotaxis of Escherichia coli to the microbiota metabolite indole. Proc. Natl. Acad. Sci. U. S. A. 117, 6114-6120. doi: 10.1073/ pnas. 1916974117

Yao, F., Jia, R., Huang, H., Yu, Y., Mei, L., Bai, L., et al. (2019). Effect of Lactobacillus paracasei N1115 and fructooligosaccharides in nonalcoholic fatty liver disease. Arch. Med. Sci. 15, 1336-1344. doi: 10.5114/aoms.2019.86611

Younes, R., and Bugianesi, E. (2019). NASH in lean individuals. Semin. Liver Dis. 39, 86-95. doi: 10.1055/s-0038-1677517

Younossi, Z. M. (2019). Non-alcoholic fatty liver disease: a global public health perspective. J. Hepatol. 70, 531-544. doi: 10.1016/j.jhep.2018.10.033

Younossi, Z. M., Koenig, A. B., Abdelatif, D., Fazel, Y., Henry, L., and Wymer, M. (2016). Global epidemiology of nonalcoholic fatty liver disease-meta-analytic assessment of prevalence, incidence, and outcomes. Hepatology 64, 73-84. doi: 10.1002/hep.28431

Younossi, Z., Tacke, F., Arrese, M., Chander Sharma, B., Mostafa, I., Bugianesi, E., et al. (2019). Global perspectives on nonalcoholic fatty liver disease and nonalcoholic steatohepatitis. Hepatology 69, 2672-2682. doi: 10.1002/ hep. 30251
Yu, Q. W., Jiang, Z. Z., and Zhang, L. Y. (2018). Bile acid regulation: a novel therapeutic strategy in non-alcoholic fatty liver disease. Pharmacol. Ther. 190, 81-90. doi: 10.1016/j.pharmthera.2018.04.005

Yuan, J., Chen, C., Cui, J., Lu, J., Yan, C., Wei, X., et al. (2019). Fatty liver disease caused by high-alcohol-producing Klebsiella pneumoniae. Cell Metab. 30, 675.e677-688.e677. doi: 10.1016/j.cmet.2019.08.018

Zeng, H., Liu, J., Jackson, M. I., Zhao, F. Q., Yan, L., and Combs, G. F. Jr. (2013). Fatty liver accompanies an increase in lactobacillus species in the hind gut of C57BL/6 mice fed a high-fat diet. J. Nutr. 143, 627-631. doi: $10.3945 /$ jn. 112.172460

Zhang, X., Coker, O. O., Chu, E. S., Fu, K., Lau, H. C. H., Wang, Y. X., et al. (2021). Dietary cholesterol drives fatty liver-associated liver cancer by modulating gut microbiota and metabolites. Gut 70, 761-774. doi: 10.1136/ gutjnl-2019-319664

Zhao, S., Jang, C., Liu, J., Uehara, K., Gilbert, M., Izzo, L., et al. (2020). Dietary fructose feeds hepatic lipogenesis via microbiota-derived acetate. Nature 579, 586-591. doi: 10.1038/s41586-020-2101-7

Zhernakova, A., Kurilshikov, A., Bonder, M. J., Tigchelaar, E. F., Schirmer, M., Vatanen, T., et al. (2016). Population-based metagenomics analysis reveals markers for gut microbiome composition and diversity. Science 352, 565-569. doi: 10.1126/science.aad3369

Zhou, D., Pan, Q., Liu, X. L., Yang, R. X., Chen, Y. W., Liu, C., et al. (2017a). Clostridium butyricum B1 alleviates high-fat diet-induced steatohepatitis in mice via enterohepatic immunoregulation. J. Gastroenterol. Hepatol. 32, 1640-1648. doi: 10.1111/jgh.13742

Zhou, D., Pan, Q., Shen, F., Cao, H. X., Ding, W. J., Chen, Y. W., et al. (2017b). Total fecal microbiota transplantation alleviates high-fat diet-induced steatohepatitis in mice via beneficial regulation of gut microbiota. Sci. Rep. 7:1529. doi: 10.1038/s41598-017-01751-y

Zhu, L., Baker, S. S., Gill, C., Liu, W., Alkhouri, R., Baker, R. D., et al. (2013). Characterization of gut microbiomes in nonalcoholic steatohepatitis (NASH) patients: a connection between endogenous alcohol and NASH. Hepatology 57, 601-609. doi: 10.1002/hep.26093

Conflict of Interest: The authors declare that the research was conducted in the absence of any commercial or financial relationships that could be construed as a potential conflict of interest.

Publisher's Note: All claims expressed in this article are solely those of the authors and do not necessarily represent those of their affiliated organizations, or those of the publisher, the editors and the reviewers. Any product that may be evaluated in this article, or claim that may be made by its manufacturer, is not guaranteed or endorsed by the publisher.

Copyright (c) $2021 \mathrm{He}$, Yao, Wang, Zhang and Bai. This is an open-access article distributed under the terms of the Creative Commons Attribution License (CC BY). The use, distribution or reproduction in other forums is permitted, provided the original author(s) and the copyright owner(s) are credited and that the original publication in this journal is cited, in accordance with accepted academic practice. No use, distribution or reproduction is permitted which does not comply with these terms. 